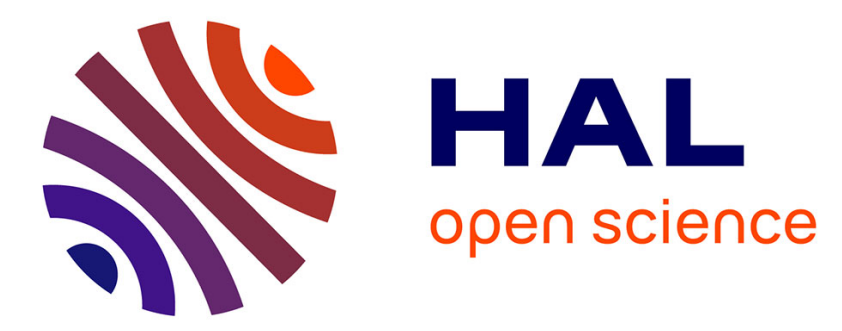

\title{
Special issue on Hybrid Systems: Computation and Control
}

Martin Fränzle, Antoine Girard, John Lygeros, Sriram Sankaranarayanan

\section{To cite this version:}

Martin Fränzle, Antoine Girard, John Lygeros, Sriram Sankaranarayanan. Special issue on Hybrid Systems: Computation and Control. Nonlinear Analysis: Hybrid Systems, 2018, 27, pp.174 - 176. 10.1016/j.nahs.2017.07.001 . hal-01620539

\section{HAL Id: hal-01620539 \\ https://hal.science/hal-01620539}

Submitted on 22 Jul 2020

HAL is a multi-disciplinary open access archive for the deposit and dissemination of scientific research documents, whether they are published or not. The documents may come from teaching and research institutions in France or abroad, or from public or private research centers.
L'archive ouverte pluridisciplinaire HAL, est destinée au dépôt et à la diffusion de documents scientifiques de niveau recherche, publiés ou non, émanant des établissements d'enseignement et de recherche français ou étrangers, des laboratoires publics ou privés. 


\section{Special issue on Hybrid Systems: Computation and Control}

\section{Overview}

This special issue contains a selection of papers originally presented at the 17th and 18th editions of the International Conference on Hybrid Systems: Computation and Control (HSCC'14 and HSCC'15).

HSCC has long been a leading, single-track conference on foundations, techniques, and tools for analysis, control, synthesis, implementation, and applications of dynamical systems that exhibit continuous and discrete (hybrid) dynamics. Applications include cyber-physical systems (CPS), mixed signal circuits, robotics, large-scale infrastructure networks, as well as natural systems such as biochemical and physiological models. In particular, HSCC solicits theoretical as well as applied research papers that present original work combining ideas from computer science and control systems.

HSCC'14 was held in Berlin, April 14-17, 2014, as part of the 7th CPSWeek (Cyber Physical Systems Week) that in addition to HSCC comprised the Real-Time and Embedded Technology and Applications Symposium (RTAS), the International Conference on Information Processing in Sensor Networks (IPSN), the International Conference on Cyber-Physical Systems (ICCPS) and the Conference on High Confidence Networked Systems (HiCoNS). About 100 participants registered for the HSCC conference. The program of the conference, chaired by Martin Fränzle and John Lygeros, consisted of the presentation of 29 regular papers, 2 tool papers, 1 case study paper, 1 keynote lecture by Gregory Batt of the Institut National de Recherche en Informatique et Automatique (INRIA) on the topic of "Cells driven by computers: long-term model predictive control of gene expression in yeast" and a panel discussion on "The Role of Robustness in Hybrid Systems". Additionally, a total of 13 posters and demos were accepted by HSCC'14 for presentation at the joint CPSWeek poster and demo session.

HSCC'15 was held in Seattle, April 13-16, 2015, alongside RTAS, IPSN and ICCPS, as part of the 8th CPSWeek. About 120 participants registered 
for the HSCC conference. The program of the conference, chaired by Antoine Girard and Sriram Sankaranarayanan, consisted of the presentation of 26 regular papers, 2 tool papers and 2 case study papers, 1 keynote lecture by Jyotirmoy V. Deshmukh from Toyota on the question "Will future cars have formally verified powertrain control software?". Additionally, a total of 11 posters and 6 demos were accepted by HSCC' 15 for presentation at the joint CPSWeek poster and demo session.

The selection of papers for this special issue was made based on the reviewer evaluations of the conference papers, on the guest editors assessment and with the coverage objective of reflecting the diversity of the conference. The authors were asked to extend their papers to include new material with respect to the conference paper. These extended papers went through the standard review process of Nonlinear Analysis: Hybrid Systems, handled by the guest editors. At the end of the review process, ten papers were accepted for publication in the special issue. They cover the whole range of topics that touch upon hybrid systems, bridging control theory on the one end (for example, stability conditions for switched and networked control systems) to theoretical computer science on the other (for example, abstraction, verification and synthesis of hybrid systems). The issue also includes a paper on an exciting application of hybrid systems theory to bipedal locomotion. A short overview of the individual papers of the special issue is included below.

\section{Papers in special issue}

\section{1. "Deciding the boundedness and dead-beat stability of constrained switch-} ing systems" by M. Philippe, G. Millerioux and R.M. Jungers

In this paper, the authors investigate two problems related to stability analysis of discrete-time linear switched systems where switching signals are constrained by some finite transition graph. Sufficient conditions are established for boundedness of trajectories of constrained switched systems, when the constrained joint spectral radius of the system matrices is equal to one. An algorithm to check these conditions is provided. Then, dead-beat stability (i.e. finite time convergence to zero) is investigated and a polynomial time algorithm is provided for verifying this property. 
2.2. "Computing compositional proofs of Input-to-Output Stability using SOS optimization and $\delta$-decidability" by A. Murthy, Md. A. Islam, S.A. Smolka and R. Grosu

In this paper, the authors compute bisimulation functions to characterize input-to-output stability of systems through the combined use of Sum-OfSquares programming and delta-decision procedures. The resulting bisimulation functions are used in conjunction with a small-gain theorem to replace components in a feedback composed system using their bounded error abstraction for the purposes of verification. The authors apply their framework on a bounded error cardiac myocyte model to replace a four variable model safely by a new model with a single variable, while proving bounded error guarantees. Extensions to non-polynomial vector fields are also presented.

\section{3. "Finite abstraction of mixed monotone systems with discrete and con- tinuous inputs" by S. Coogan and M. Arcak}

In this paper, the authors propose an efficient abstraction technique for a class of discrete-time nonlinear systems. The considered class is that of mixed monotone systems, an extension of the class of usual monotone systems. For such systems, the reachable set from a rectangular set can be over-approximated by evaluating a decomposition function at only two points. This property makes it possible to compute discrete abstractions of mixed monotone systems in a simple and efficient way. Both the case of discrete and continuous inputs are considered. Case studies in insect population dynamics or vehicular traffic networks are reported.

2.4. "Temporal logic control for stochastic linear systems using abstraction refinement of probabilistic games" by $M$. Svoreňová, J. Křetínský, $M$. Chmelik, K. Chatterjee, I. Černá and C. Belta

In this paper, the authors consider the control of discrete-time, stochastic linear dynamical systems for temporal logic objectives written in the generalized reactivity $(\mathrm{GR}(1))$ class of formulas. They provide an iterative approach that partially computes the set of winning initial states for the specification after any finite number of iterations. Further, upon termination, their procedure is guaranteed to yield the full set. The complexity of the algorithm is characterized. For the special case of reachability properties, a more efficient algorithm is provided. The algorithms are demonstrated on an illustrative case study. 


\section{5. "Bounded invariant verification for time-delayed nonlinear networked dynamical systems" by Z. Huang, C. Fan and S. Mitra}

This article deals with the problem of verifying bounded invariant for dynamical systems featuring delayed interconnections, as found for example in networked control systems. It employs a notion of input-to-state discrepancy

to generate a low dimensional system whose trajectories are bounding the distance between the trajectories of a complex network that may arise under changed initial states. This facilitates a sound and relatively complete algorithm for bounded invariant verification of networked nonlinear dynamical systems interacting through possibly delayed signals.

\section{6. "On stability of discrete-time switched systems" by A. Kundu and D. Chatterjee}

This paper deals with stability conditions, primarily for switched nonlinear, discrete time systems. The discrete time switched systems considered are such that any point in time their state evolves according to dynamics selected among a finite family of transition functions, with the switching among the different possible dynamics controlled through a so-called switching signal. The authors first establish conditions on the switching signals that ensure global asymptotic stability to the zero state. In the spirit of dwell time results, the conditions are based on growth relations among multiple Lyapunov functions; later in the paper these conditions are also extended to a class of switching systems in continuous time. Based on these conditions, a systematic procedure for constructing discrete time switching sequences that, in a sense, globally asymptotically stabilise the system is then developed. The results are finally extended to systems with inputs in the sense of input-to-state stability and demonstrated on a small, benchmark example.

2.7. "Tradeoffs between quality-of-control and quality-of-service in large-scale nonlinear networked control systems" by D.P. Borgers, R. Geiselhart and W.P.M.H. Heemels

This paper investigates the properties of controlled dynamical systems where a communication network is included in the feedback loop and used to carry sensing and actuation information. The goal is to quantify the relation between the quality of service guarantees provided by the communication network and the performance of the closed loop system. In this paper quality of service is quantified through the maximum allowable transmission interval (a guarantee on the maximum time between two consecutive 
transmissions) and the maximum allowable delay (a similar guarantee on the maximum time between the transmission and reception of information). Control performance on the other hand is characterised through guarantees of input-to-state stability and the corresponding convergence rates; as usual a multiple Lyapunov function approach is adopted for this characterisation. The paper develops precise conditions on the effect of quality of service on stability, allowing one to determine bounds on the possible degradation of control performance if the quality of service deteriorates. The analysis is carried out in continuous time, starting with the very general framework of hybrid differential inclusions.

\section{8. "Model measuring for discrete and hybrid systems" by T.A. Henzinger and J. Otop}

Within this article, the authors address the problem of measuring a model in the sense of determining the maximum permissible amount of perturbation applicable to constants occurring in the model that would guarantee preservation of satisfaction of its specification. This generalization of traditional Boolean verification is pursued for a broad set of models, ranging from weighted word and tree automata to parametric monotonic hybrid automata. As model measuring my be applied to systems not satisfying their specification, in which case model measuring would determine the minimum distance to a model variant satisfying the specification, the related question of model repair towards requirements satisfaction is also investigated.

\section{9. "First steps toward formal controller synthesis for bipedal robots with experimental implementation" by A.D. Ames, P. Tabuada, A. Jones, W.-L. Ma, M. Rungger, B. Schrmann, S. Kolathaya and J.W. Grizzle}

In this paper, the authors apply formal methods to controller synthesis for bipedal robots. The control synthesis approach consists of two steps. Firstly, traditional nonlinear control techniques are used to stabilize the system on a low dimensional manifold; the evolution of the system on this manifold is described by the hybrid zero dynamics. Secondly, abstraction based control is applied to the hybrid zero dynamics to enforce safety specifications to be satisfied by the original system. The approach is validated using numerical simulations. Experimental results obtained by implementing the synthesized controllers on a bipedal robot are promising and show the relevance of the formal methods in control applications. 
2.10. "A Type-based analysis of causality loops in hybrid systems modelers" by A. Benveniste, T. Bourke, B. Caillaud, B. Pagano and M. Pouzet

This article addresses the static detection of causality loops in hybrid system models. It establishes a type system expressing dependencies between signals and enforcing absence of causality loops as a necessary condition for a system being well-typed. This ensures their causal consistency as necessary for avoiding deadlock situations during sequential simulation and for generating statically scheduled code.

\section{Martin Fränzle \\ Department of Computing Science Carl von Ossietzky Universität}

Ammerlnder Heerstr. 114-118, 26129 Oldenburg, Germany Email adress: martin.fraenzle@informatik.uni-oldenburg.de

Antoine Girard Laboratoire des Signaux et Systèmes CNRS, CentraleSupélec, Univ. Paris-Sud, Univ. Paris-Saclay 3 rue Joliot Curie, 91192 Gif-sur-Yvette, France Email adress: antoine.girard@12s.centralesupelec.fr

John Lygeros Automatic Control Laboratory ETH Zurich Physikstrasse 3 CH-8092 Zurich, Switzerland Email adress: jlygeros@ethz.ch

Sriram Sankaranarayanan Department of Computer Science University of Colorado, Boulder Boulder, CO, 80301, USA Email address: srirams@colorado.edu 